\title{
High prevalence of SMARCB1 constitutional abnormalities including mosaicism in malignant rhabdoid tumors
}

\author{
Ryota Shirai ${ }^{1,2} \cdot{\text { Tomoo Osumi } \mathbb{1}^{1,3} \cdot \text { Keita Terashima }^{3} \cdot \text { Chikako Kiyotani }^{3} \cdot \text { Meri Uchiyama }^{1,3} \cdot \text { Shinichi Tsujimoto }}^{1,3}$. \\ Masanori Yoshida ${ }^{1,2} \cdot$ Kaoru Yoshida $^{1} \cdot$ Toru Uchiyama $^{4} \cdot$ Daisuke Tomizawa $^{3} \cdot$ Yoko Shioda $^{3} \cdot$ Masahiro Sekiguchi $^{5}$. \\ Kenichiro Watanabe ${ }^{6} \cdot$ Dai Keino $^{7} \cdot$ Hitomi Ueno-Yokohata $^{1} \cdot$ Kentaro Ohki $^{1} \cdot$ Junko Takita $^{5}$ - Shuichi Ito ${ }^{2}$. \\ Takao Deguchi $^{3} \cdot$ Nobutaka Kiyokawa $^{1} \cdot$ Hideki Ogiwara $^{8} \cdot$ Tomoro Hishiki $^{9} \cdot$ Seishi Ogawa ${ }^{10} \cdot$ Hajime Okita $^{11,12}$. \\ Kimikazu Matsumoto ${ }^{3} \cdot$ Takako Yoshioka $^{11} \cdot$ Motohiro Kato ${ }^{1,3}$
}

Received: 22 November 2019 / Revised: 2 March 2020 / Accepted: 10 March 2020 / Published online: 26 March 2020

(c) European Society of Human Genetics 2020

\begin{abstract}
Intensive analysis of the SMARCBI gene in malignant rhabdoid tumors (MRT) revealed eight of 16 patients with constitutional genetic variants. Three patients had mosaicism of deletion/variant of the SMARCB1 gene, which conventional methods might overlook. The prevalence of cancer predisposition in MRT may thus be higher than previously reported.
\end{abstract}

\section{Background}

Malignant rhabdoid tumor (MRT), including atypical teratoid/rhabdoid tumor (AT/RT), MRT of the kidney, and extracranial MRT, is a rare and highly aggressive malignancy. These three subtypes have a common genetic hallmark, namely, loss-of-function variants or deletions in the SMARCB1 (also known as the hSNF5/INI1/BAF47) gene [1]. Most MRT cells show bi-allelic alteration in

Supplementary information The online version of this article (https:// doi.org/10.1038/s41431-020-0614-z) contains supplementary material, which is available to authorized users.

Motohiro Kato

katom-tky@umin.ac.jp

1 Department of Pediatric Hematology and Oncology Research, National Research Institute for Child Health and Development, Tokyo, Japan

2 Department of Pediatrics, Yokohama City University, Yokohama, Japan

3 Children's Cancer Center, National Center for Child Health and Development, Tokyo, Japan

4 Department of Human Genetics, National Research Institute for Child Health and Development, Tokyo, Japan

5 Department of Pediatrics, University of Tokyo, Tokyo, Japan

6 Department of Hematology and Oncology, Shizuoka Children's Hospital, Shizuoka, Japan
SMARCB1 [1] and are a typical example of Knudson's twohit hypothesis [2-4].

Variants in the SMARCB1 gene are not always somatically acquired. Previous studies demonstrated that approximately one-third of patients had a germline SMARCBI abnormality resulting in the first hit of Rhabdoid Tumor Predisposition Syndrome (RTPS) [5-7]. Actually, most germline SMARCB1 abnormalities are presumed to be sporadic, but a few familial cases have been reported [5, 6, 8]. Although some studies reported that individuals with RTPS showed a high rate of multiple tumor development at an early age, the clinical characteristics and prognosis remain controversial $[6,7,9]$.

In a study by Acuna-Hidalgo et al., intensive genetic analysis revealed that some variants which were previously

7 Department of Pediatrics, St. Marianna University School of Medicine Hospital, Kawasaki, Japan

8 Division of Neurosurgery, National Center for Child Health and Development, Tokyo, Japan

9 Division of Surgery Oncology, Children's Cancer Center, National Center for Child Health and Development, Tokyo, Japan

10 Department of Pathology and Tumor Biology, Graduate School of Medicine Kyoto University, Kyoto, Japan

11 Department of Pathology, National Center for Child Health and Development, Tokyo, Japan

12 Department of Pathology, Keio University School of Medicine, Tokyo, Japan 
considered to be de novo actually were post-zygotic mosaic variants with a proportion of $6.5 \%$ [10], and it has been suggested that similar mosaic variants may be present in a certain fraction of childhood cancer cases [11]. However, no studies to date have focused on the frequency of post-zygotic mosaicism in MRT. We investigated herein the prevalence of germline variants in the SMARCB1 gene by intensive analysis, including quantitative assessment of copy numbers and a variant detection assay using droplet digital PCR (ddPCR) capable of detecting low-frequency germline alterations.

\section{Methods}

\section{Patients}

This study was approved by the ethics committee at the National Center for Child Health and Development (\#1035) and written informed consent was obtained from the patients and/or guardians. The enrolled patients received the diagnosis of MRT at the National Center for Child Health and Development, the Shizuoka Children's Hospital or St. Marianna University School of Medicine Hospital between 2006 and 2018. Histological diagnosis was confirmed by the negativity of SMARCB1 by immunohistochemistry. In total, 16 patients were included. Both tumor-derived DNA and germline DNA were obtained from all patients (Table 1).

\section{DNA isolation and PCR analyses for SMARCB1}

DNA was extracted using a QIAamp DNA Mini kit or a QIAamp DNA FFPE tissue kit (Quiagen, Hilden, Germany) following the manufacturer's instructions. Standard PCR reactions were carried out using AmpliTaq Gold 360 Master mix (Applied Biosystems, Carlsbad, CA, USA). The primer sequences for each exon of SMARCB1 are shown in Supplementary Table 1.

First, screening for SMARCBI alterations in the tumor specimens was done by direct sequencing and ddPCR in all the samples. SNP array analysis was additionally performed for 12 cases with a sufficient quantity and quality of DNA. Then, analysis of germline DNA samples focusing on the genomic abnormalities detected in the paired tumors in each case was performed.

\section{Droplet digital PCR}

The ddPCR analysis was performed using QX-200 (Bio-rad, Hercules, CA, USA) to determine the copy number of each exon of SMARCBI (exons are numbered like in NM_003073.3) [12]. Specific primers and probes were designed for each exon (Supplementary Table 2). An RPP30 gene primer/probe mix (Bio-Rad, Hercules, CA,
USA) was used as an internal control [13]. Sensitivity testing of copy number analysis for exon 4 of SMARCB1 showed that the detection limit for deletions was as low as 0.75\% (Supplementary Fig. 1). Primer/probe sets were also designed for single nucleotide variants and indels detected in tumors by direct sequencing and were used in analyzing for the presence of low-frequency germline abnormalities (Supplementary Table 3).

\section{SNP array analysis}

Genome-wide analysis for copy number alterations and allelic imbalances was performed by single nucleotide polymorphism (SNP) array analysis. DNA extracted from the samples was analyzed using GeneChip Human Mapping $250 \mathrm{~K} \mathrm{NspI}$ array (Affymetrix, Santa Clara, CA, USA). The obtained data were processed using CNAG/AsCNAR software according to the previously described method [14].

\section{Statistical analyses}

The difference in the median age between patient groups was analyzed by variant status using the Mann-Whitney $U$ test. Overall survival and progression-free survival (PFS) were estimated using the Kaplan-Meier method and compared using the log-rank test. Cox regression models were used to examined the prognostic factors affecting survival time.

\section{Results}

The 16 patients included those with AT/RT $(n=8)$, MRT of the kidney $(n=2)$, extracranial MRT $(n=4)$, or both intracranial and extracranial MRT $(n=2)$. The median age at diagnosis was 16.5 months (range, 0-154 months). Bi-allelic alterations of the SMARCB1 gene were found in the tumor cells of all 16 patients (Table 1). Of the 32 genomic alterations, ten were nonsynonymous nucleotide substitutions and frameshift variants, ten were deletions of one or more exons within SMARCB1, and 12 were copy number neutral losses of heterozygosity identified mainly by SNP array analysis. All the detected nonsynonymous variants were nonsense variants or frameshift insertions/ deletions leading to the early truncation of the SMARCB1 protein (Table 1 and Supplementary Fig. 2). No patients had any malformations or neurodevelopmental disorders.

In eight of 16 cases (50\%), genomic alterations observed in the tumor-derived DNA were also detected in constitutional DNA. It is worth noting that three patients had germline mosaicism (UPN4, 5 and 8) (Fig. 1 and Supplementary Fig. 3). Two patients had mosaic deletion, including $S M A R C B 1$, and the average copy number of the deleted region in the SMARCB1 gene in the germline was 1.60 


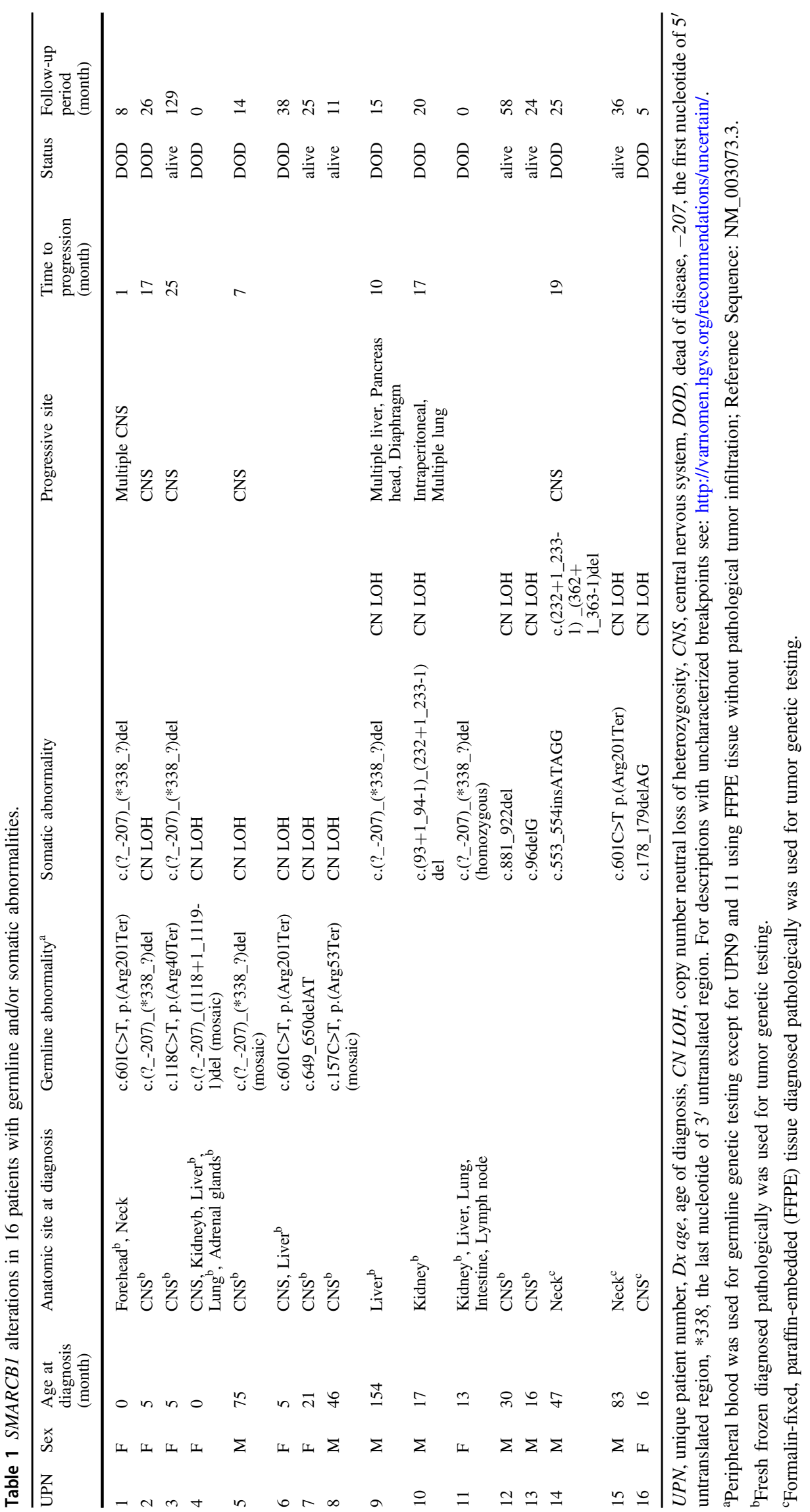


A
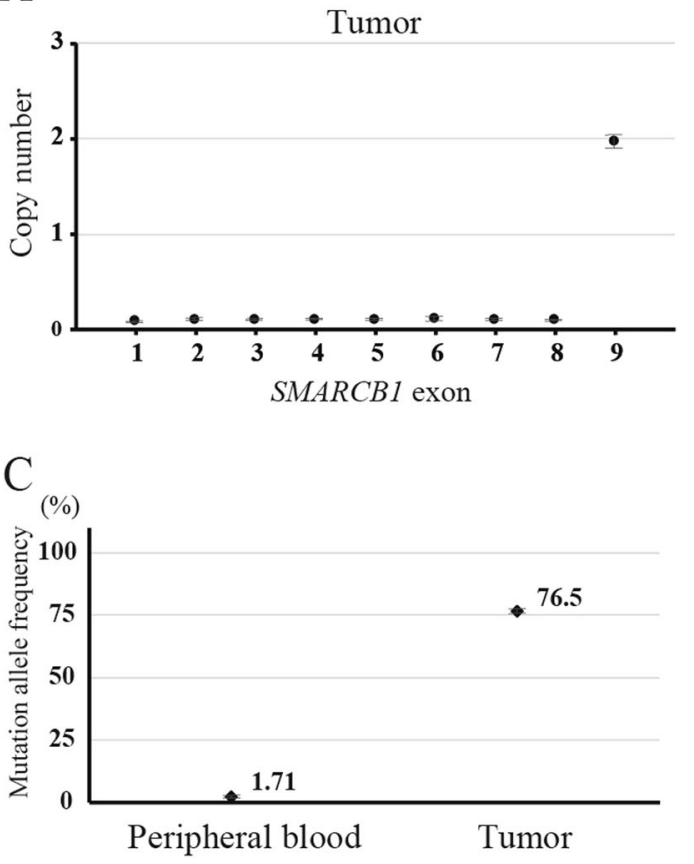

Fig. 1 Representative results of mosaic alterations in SMARCB1. a, b Shown are the copy number analyses for each exon of the SMARCB1 gene using digital droplet PCR. Homozygous deletion of exons 1-8 was observed in tumor specimens of UPN4 (a). The copy number of exons 1-8 was estimated at 1.60 , indicating that $40 \%$ of cells in

(UPN4) and 1.76 (UPN5), respectively, indicating that 40 and $24 \%$ of their cells had heterozygous deletions. For UPN8, the fraction of SMARCB1 variants in normal cells was $1.7 \%$, indicating that $3.4 \%$ of cells had the SMARCBI variant.

The median age at diagnosis with or without germline abnormalities was 5 months (range, 0-75 months) and 23.5 months (range, 13-154 months), for the respective groups, and the age difference was not statistically significant ( $p=0.082$, Supplementary Fig. 4 ). Two cases with both intracranial and extracranial MRT showed constitutional genomic alterations. Recurrence with or without constitutional abnormalities was observed in four and three patients, respectively, in whom PFS at 2 years was $46.9 \%$ and $37.5 \%$ ( $p=0.52$, Supplementary Fig. 5).

In three patients (UPN1, 2, and 4) with a constitutional abnormality, genetic analysis of their parents was performed using peripheral blood samples following genetic counseling. None of the parents had any abnormality of the SMARCB1 gene observed in their offspring.

\section{Discussion}

We performed a detailed analysis of the prevalence of SMARCB1 germline/constitutional abnormalities using the ddPCR assay, and identified three new cases (37.5\%) among the eight patients with a germline abnormality with mosaicism
B

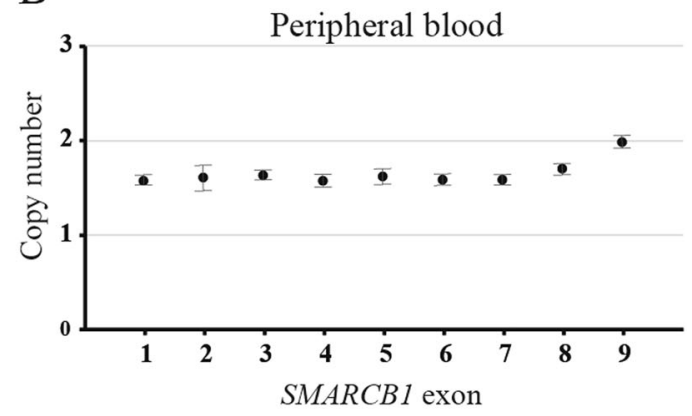

peripheral blood had a heterozygous deletion in this region (b). c A nonsense variant $(c .157 \mathrm{C}>\mathrm{T})$ was observed in $76.5 \%$ of tumor cells in UPN8 and detected in peripheral blood at a mutant allele frequency of $1.71 \%$ indicating a mosaicism rate of $3.42 \%$. Error bars represent $95 \%$ confidence intervals.

contributing to the pathogenesis of MRT. These cases had a small proportion of mutated cells indicative of mosaicism and were therefore possibly overlooked by conventional qualitative analyses. Our study revealed a prevalence of RTPS in almost half of the MRT cases exceeding previously reported figures [5-7] and underscoring the importance of constitutional alterations in the etiology of pediatric cancers, as shown in recent, large-scale studies $[15,16]$.

In our cohort, truncating SMARCBI variants, which predispose to RTPS, were observed in all eight patients with constitutional abnormalities including mosaicism [17]. Germline variants in SMARCB1 are also known as causes of schwannomatosis and Coffin-Siris syndrome, thus some patients may have manifestations of these diseases, as well as of RTPS, although none of our cases had the typical phenotype of schwannomatosis and Coffin-Siris syndrome during follow-up period [12, 17].

Some patients with RTPS show MRT development at an older age. In our cohort, two (UPN5 and 8) of eight patients with RTPS had MRT onset at age 2 years or older, suggesting that genetic screening tests are needed in all patients regardless of age. Interestingly, the two cases showed a low frequency of mosaicism for SMARCBI variants.

As in previous reports [5-7], most patients with multiple lesions had germline abnormalities. Bhatt et al. reported MRT development in the soft tissue of a patient with a germline SMARCB1 variant 8 years after the end of AT/RT 
treatment [18]. Germline carriers are presumably at risk of second MRT development, which is difficult to distinguish from a recurrence.

Considering the high frequency of patients with RTPS, genetic counseling for parents is necessary because familial cases have been reported despite their low frequency $[5,6,8,9,19,20]$. Although genetic tests of the parents (UPN1, 2, and 4) were all negative, the possibility of gonadal mosaicism should be considered because there are patients with constitutional mosaicism and also previous reported cases $[5,6,8,9,19,20]$.

This study has several limitations. First, due to the small sample size, the precise prevalence of germline variants and the correlation between clinical features and variant origins could not be accurately assessed and require investigation in largerscale studies. Second, although our ddPCR method was able to detect a frequency of mosaicism as low as $1 \%$, the prevalence of constitutional variants may have been underestimated due to overlooking other, lower frequency mosaicisms.

In conclusion, approximately half the MRT cases in this study had SMARCBI constitutional alterations, including low-frequency mosaicism of deletion or nonsynonymous variants. Considering the presence of low-frequency mosaicisms, inherited germline variants in predisposition genes are more important than previously assumed for the pathogenesis of pediatric cancers.

Acknowledgements The authors would like to thank Ms Miwako Tatsuno, Ms Shinobu Kobayashi and Ms Etsuko Mochizuki for their technical assistance. We also thank Mr Yukinori Yatsuda (Bio-rad) for his assistance with the ddPCR analysis. The authors thank the staff of the Center for Clinical Research and Development at NCCHD for their editorial support.

Funding This work was supported in part by a grant from the National Center for Child Health and Development (grant number 2019B-8) and by the Japan Agency for Medical Research and development (AMED) (grant number JP19ck0106467 and JP19cm0106509).

\section{Compliance with ethical standards}

Conflict of interest The authors declare that they have no conflict of interest.

Publisher's note Springer Nature remains neutral with regard to jurisdictional claims in published maps and institutional affiliations.

\section{References}

1. Versteege I, Sevenet N, Lange J, Rousseau-Merck MF, Ambros P, Handgretinger R, et al. Truncating mutations of hSNF5/INI1 in aggressive paediatric cancer. Nature. 1998;394:203-6.

2. Reisman D, Glaros S, Thompson EA. The SWI/SNF complex and cancer. Oncogene. 2009;28:1653-68.
3. Roberts CW, Biegel JA. The role of SMARCB1/INI1 in development of rhabdoid tumor. Cancer Biol Ther. 2009;8:412-6.

4. Knudson AG Jr. Mutation and cancer: statistical study of retinoblastoma. Proc Natl Acad Sci USA. 1971;68:820-3.

5. Bourdeaut F, Lequin D, Brugieres L, Reynaud S, Dufour C, Doz F, et al. Frequent hSNF5/INI1 germline mutations in patients with rhabdoid tumor. Clin Cancer Res. 2011;17:31-8.

6. Eaton KW, Tooke LS, Wainwright LM, Judkins AR, Biegel JA. Spectrum of SMARCB1/INI1 mutations in familial and sporadic rhabdoid tumors. Pediatr Blood Cancer. 2011;56:7-15.

7. Kordes U, Gesk S, Fruhwald MC, Graf N, Leuschner I, Hasselblatt $\mathrm{M}$, et al. Clinical and molecular features in patients with atypical teratoid rhabdoid tumor or malignant rhabdoid tumor. Genes Chromosomes Cancer. 2010;49:176-81.

8. Sevenet N, Sheridan E, Amram D, Schneider P, Handgretinger R, Delattre O. Constitutional mutations of the hSNF5/INI1 gene predispose to a variety of cancers. Am J Hum Genet. 1999;65:1342-8.

9. Bruggers CS, Bleyl SB, Pysher T, Barnette P, Afify Z, Walker M, et al. Clinicopathologic comparison of familial versus sporadic atypical teratoid/rhabdoid tumors (AT/RT) of the central nervous system. Pediatr Blood Cancer. 2011;56:1026-31.

10. Acuna-Hidalgo R, Bo T, Kwint MP, van de Vorst M, Pinelli M, Veltman JA, et al. Post-zygotic point mutations are an underrecognized source of de novo genomic variation. Am J Hum Genet. 2015;97:67-74.

11. Renaux-Petel M, Charbonnier F, Thery JC, Fermey P, Lienard G, Bou J, et al. Contribution of de novo and mosaic TP53 mutations to Li-Fraumeni syndrome. J Med Genet. 2018;55:173-80.

12. Holsten T, Bens S, Oyen F, Nemes K, Hasselblatt M, Kordes U, et al. Germline variants in SMARCB1 and other members of the BAF chromatin-remodeling complex across human disease entities: a meta-analysis. Eur J Hum Genet. 2018;26:1083-93.

13. Kluwe L. Digital PCR for discriminating mosaic deletions and for determining proportion of tumor cells in specimen. Eur $\mathbf{J}$ Hum Genet. 2016;24:1644.

14. Yamamoto G, Nannya Y, Kato M, Sanada M, Levine RL, Kawamata N, et al. Highly sensitive method for genomewide detection of allelic composition in nonpaired, primary tumor specimens by use of affymetrix single-nucleotide-polymorphism genotyping microarrays. Am J Hum Genet. 2007;81:114-26.

15. Zhang J, Walsh MF, Wu G, Edmonson MN, Gruber TA, Easton J, et al. Germline mutations in predisposition genes in pediatric cancer. N Engl J Med. 2015;373:2336-46.

16. Gröbner SN, Worst BC, Weischenfeldt J, Buchhalter I, Kleinheinz $\mathrm{K}$, Rudneva VA, et al. The landscape of genomic alterations across childhood cancers. Nature. 2018;555:321.

17. Smith MJ, Wallace AJ, Bowers NL, Eaton H, Evans DGR. SMARCB1 mutations in schwannomatosis and genotype correlations with rhabdoid tumors. Cancer Genet. 2014;207:373-8.

18. Bhatt MD, Al-Karmi S, Druker H, Gupta A, Lu M, Malkin D, et al. Second rhabdoid tumor 8 years after treatment of atypical teratoid/rhabdoid tumor in a child with germline SMARCB1 mutation. Pediatr Blood Cancer. 2019;66:e27546.

19. Janson K, Nedzi LA, David O, Schorin M, Walsh JW, Bhattacharjee $\mathrm{M}$, et al. Predisposition to atypical teratoid/rhabdoid tumor due to an inherited INI1 mutation. Pediatr Blood Cancer. 2006;47:279-84.

20. Gigante L, Paganini I, Frontali M, Ciabattoni S, Sangiuolo FC, Papi L. Rhabdoid tumor predisposition syndrome caused by SMARCB1 constitutional deletion: prenatal detection of new case of recurrence in siblings due to gonadal mosaicism. Fam Cancer. 2016;15:123-6. 